\title{
Outcome of Graves' Disease Treated with Anti-Thyroid Drugs and Time Courses of Anti-Tsh Receptor Antibodies
}

\author{
Toshihide Yamamoto* \\ Yao Tokushukai General Hospital, Yao, Osaka, Japan
}

*Corresponding author: Toshihide Yamamoto, Yao Tokushukai General Hospital, Yao, Osaka, Japan, Tel: 8172-993-8501; E-mail: toshihide.yamamoto@tokushukai.jp

Received: 15 Apr, 2019 | Accepted: 26 Apr, 2019 | Published: 30 Apr, 2019

Citation: Yamamoto T (2019) Outcome of Graves' disease Treated with Anti-Thyroid Drugs and Time Courses of Anti-Tsh Receptor Antibodies. Int J Endocrinol Metab Disord 5(1): dx.doi.org/10.16966/2380-548X.155

Copyright: (c) 2019 Yamamoto T. This is an open-access article distributed under the terms of the Creative Commons Attribution License, which permits unrestricted use, distribution, and reproduction in any medium, provided the original author and source are credited.

\begin{abstract}
Objective: Remission Rate of Graves' disease (GD) by Anti-thyroid Drugs (ATD) remains around $50 \%$ and renovated ATD is not expected soon. As GD is an autoimmune disease associated with Anti-TSH receptor Auto-antibodies (TRAb) and blood TRAb levels presumably reflect immune dysregulation, better practice of ATD therapy could be worked out using TRAb.

Methods: 101 patients were treated with ATD in a titration regimen. Their serum TRAb levels were serially measured by a $2^{\text {nd }}$ generation assay (DYNO test TRAK ${ }^{\text {TM }}$ human kit), henceforth designated as hTRAb levels. ATD was continued until the hTRAb levels become undetectable ( $<1.0$ IU/L) consecutively twice (Target A) or reducing in gray-zone (1.0-1.5 IU/L) (Target B). Remission rates, defined by no relapse within-one-year postATD, were assessed in patients having attained Target A or Target B. Time Courses (TCs) of hTRAb levels were drawn. Relations of TC patterns and outcomes were sought.

Results: The relapse-free remission rate of patients having attained Target $\mathrm{A}(\mathrm{N}=41)$ among those having attained Target $\mathrm{A}$ with or without relapse $(\mathrm{N}=52)$ was $78.8 \%$. The relapse-free remission rate of patients having attained either Target $A$ or Target $B(N=52)$ in the total patients was $51.5 \%$. Remission was associated with four TC patterns; throughout undetectable levels, gradual reducing and converging to undetectable levels twice, reducing and converging in gray-zone levels, and one or two flair-ups in gradually reducing levels.
\end{abstract}

Conclusion: Until better biomarkers reflecting altered immune status become available, outcomes of GD can be predicted by serially measured TRAb levels during ATD treatment.

Keywords: Graves' disease; Remission rate; Anti-thyroid drug; Anti-thyrotropin receptor auto-antibodies

\section{Background}

Anti-Thyroid Drugs (ATDs), introduced for treatment of hyperthyroidism by Edwin Astwood three quarters of a century ago [1], have been employed for management of Graves' Disease (GD) worldwide. However, remission of GD through ATD treatment is still not satisfactory, e.g., a recent meta-analysis reported there were 3696 relapses in 7595 patients (48.7\%) after ATD withdrawal [2]. As new ATDs do not appear to be available in the near future, we have to work out better practice of ATD administration and withdrawal to improve the remission rate.

As GD is an autoimmune disease caused by TRAb, blood TRAb levels, provided reflecting the status of immune dysregulation, could be used to predict remission during ATD treatment. The American Thyroid Association (ATA) made the following recommendations concerning ATD withdrawal in patients being kept on prolonged ATD treatment: Anti-Thyrotropin Receptor Autoantibodies (TRAb) assessment at the end of the course of ATD therapy is useful to discern prospect of remission or non-remission; TRAb might be monitored every 1-2 years; and ATD discontinuation is considered if TRAb become negative over long follow-up period [3]. The $2^{\text {nd }}$ generation assays of TRAb are sufficiently sensitive to diagnose GD $[4,5]$. The assays are competition-based assays and do not differentiate three kinds of TRAb, i.e., receptor-blocking, receptor-stimulating, and receptor-neutral TRAb [6]. Therefore, TRAb levels of active GD patients, at least certain fractions of them, are presumed to show stimulating activity while undetectable TRAb levels of patients in remission presumed to show disappearance of stimulating activity.

With the $2^{\text {nd }}$ generation assays, the relation of TRAb levels below cutoff levels prior to ATD withdrawal were investigated in several studies. Six studies were selected, in which studies were performed in following designs; TRAb levels measured by the same $2^{\text {nd }}$ generation assay as used in the present study, cutoff levels of TRAb set as 1.5 IU/L or less, numbers of patients $>20$, follow-up periods after ATD 
withdrawal for 1-4 years [7-13]. The relapse rates of these studies varied from $26.2 \%$ [9] to 58\% [12]. Liu L, et al. [14] treated $306 \mathrm{GD}$ patients with ATD for 18 months in a titration regimen (block and replacement regimen for patients having become hypothyroid) and observed remission in 141 patients (46.1\%) having achieved TRAb levels (measured by another $2^{\text {nd }}$ generation assay) below cutoff level of $14 \mathrm{IU} / \mathrm{L}$. Faced these figures, endocrinologists may feel hesitant about discontinuing ATD in patients with GD if they have persistently maintained euthyroidism with a minimal dose of ATD. The author anticipated better remission rate could be obtained if ATD is maintained until TRAb levels are undetectable on two consecutive occasions. As a byproduct of the investigations, fair amount of the data of TRAb during ATD treatment has accumulated. Using these data, the Time Courses (TCs) of TRAb levels were drawn. From the patterns of TCs, the outcomes of ATD treatment were assessed.

\section{Clinical Study}

\section{Patients and treatment protocols}

One hundred and eighteen patients were treated for GD at the author's clinic over an 11-year period starting October 2003 until March 2014 when the clinic closed. Exclusions were made for 17 patients lost to follow-up. Forty-six patients presented to the author within three months of awareness of clinical manifestations and the rest were referred by other physicians. A diagnosis of GD was based on clinical manifestations, serum thyroid hormones, TSH, and TRAb. Patients were treated with either thiamathole Mercazole $^{\mathrm{TM}}$, Asuka Pharmaceutical Co. Ltd., Tokyo) or propylthiouracil (Propacil ${ }^{\mathrm{TM}}$, Asuka Pharmaceutical Co., Ltd) in a titration regimen. The administration of ATD was discontinued when any of the following three conditions were met: Target $\mathrm{A}$, persistent euthyroidism and hTRAb levels (Cf. method section) undetectable $(<1.0 \mathrm{IU} / \mathrm{L})$ on two consecutive occasions separated by three or more months while being maintained on ATD one tablet every other day (henceforth, minimal dose); Target B, persistent euthyroidism while being maintained on minimal dose of ATD for $\geq 6$ months [15] and hTRAb levels remaining repetitively in gray-zone (1.0 1.5 IU/L) (Cf. methods of monitoring); development of persistent hypothyroidism without ATD for $\geq 3$ months. In patients whose hTRAb levels continued to fluctuate or remained above $1.5 \mathrm{IU} / \mathrm{L}$, the administration of ATD was maintained unless patients choose ablation therapy. After having attained Targets A or B, patients were followed for one year. Remission was defined by maintaining euthyroidism and no relapse within one year after ATD withdrawal (henceforth, within-one-year post-ATD relapse).

The treatment protocol was approved by an ad hoc review committee of the clinic. Patients gave their informed consent.

\section{Methods of Monitoring}

Serum levels of TRAb were measured in a $2^{\text {nd }}$ generation competitive binding assay with DYNO test TRAK ${ }^{\mathrm{TM}}$ human kit [4] (manufactured by BRAHMS AG, Berlin: imported by Yamasa Syoyu, Inc., Choshi, Chiba), henceforth, levels designated as hTRAb levels. A level of $<1.0$ IU/L was considered as negative (indicating absence of TRAb) and levels between 1.0 and 1.5 IU/L as gray-zone level [4]. All assays were performed by Special Reference Laboratories (SRL, Hachioji, Tokyo).

The hTRAb levels were measured periodically in every patient, the frequency depending on the pattern of changes. Levels were measured every 3 or 4 months in patients with declining titers, less frequently in patients with high titers, until withdrawal of ATD.

The outcomes were classified in five categories: outcome la, remission by attaining Target $\mathrm{A}$; outcome $1 \mathrm{~b}$, remission by attaining
Target B; outcome 2a, attaining neither Target A nor Target B and persistently being treated by ATD; outcome $2 \mathrm{~b}$, opting either surgery or radioiodine treatment because attaining Target A or Target B unlikely; outcome 3, developing either persistent hypothyroidism after the withdrawal of ATD or TRAb-negative hyperthyroidism. When patients relapsed having attained Target A or Target B within one year, they were treated again with ATD in the same schedule as before.

\section{Inquiry}

1. Remission rates and the duration of ATD treatment were sought in patients having achieved remission by attaining either Target $\mathrm{A}$ or Target B (prospective study).

2. Time courses (TCs) of hTRAb levels of all patients excluding those of outcome $3(\mathrm{~N}=5)$ were drawn with time in the abscissa and hTRAb titers (logarithmic scale) in the ordinate and were assembled into four outcome categories, i.e. outcomes $1 \mathrm{a}, 1 \mathrm{~b}, 2 \mathrm{a}$, and $2 \mathrm{~b}$. The relations of patterns of TCs and the outcomes were sought (retrospective study).

\section{Results}

The relapse-free remission rate of the patients attaining Target A $(\mathrm{N}=41)$ or Target $\mathrm{B}(\mathrm{N}=11)$ in the total patients was $51.4 \%(52 / 101)$. The remission rate of patients having achieved remission by Target $\mathrm{A}$ $(\mathrm{N}=41)$ in patients having attained Target $\mathrm{A}$ (with or without relapse) $(\mathrm{N}=52)$ was $78.8 \%$. Likewise, the remission rate of combined patients having achieved remission by Target $\mathrm{A}(\mathrm{N}=41)$ and those having achieved remission by Target $\mathrm{B}(\mathrm{N}=11)$ in those having attained Target A (with or without relapse) $(\mathrm{N}=52)$ and those having attained Target B $(\mathrm{N}=11)$ was $82.5 \%(52 / 63)$. It took 51.4 months (95\% confidence interval, 48.9-53.9 months) for 41 patients to achieve relapse-free remission by Target A and 74.6 months (95\% confidence interval, 42.9-106.5 months) for 11 patients to achieve relapse-free remission by Target B.

The outcomes of 101 patients are divided into five outcome categories (Table 1).

\section{Outcome 1a}

Though 52 patients attained Target A, 41 patients achieved remission and 11 patients had within-one-year post-ATD relapse. The final outcomes of the latter patients were diverse: two were re-treated and eventually achieved remission by Target $A(N=1)$ or by Target $B$ $(\mathrm{N}=1)$; one opted for surgery; four were re-treated and remained under ATD treatment at the end of this study; two developed hypothyroidism and other two remained under ATD treatment for TRAb-negative hyperthyroidism.

\section{Outcome 1b}

11 patients achieved by Target B (including one having within-oneyear post-ATD relapse).

\section{Outcomes 2a, 2b, and 3}

The outcomes of other 49 patients were either persistent active Graves' disease requiring ATD $(\mathrm{N}=38)$ (outcome 2a), ablation therapy $(\mathrm{N}=6)$ (outcome 2b), development of hypothyroidism $(\mathrm{N}=3)$ or TRAbnegative hyperthyroidism $(\mathrm{N}=2)$ (outcome 3$)$.

The TCs of 96 patients (excluding patients of outcome 3) were assembled according to the outcome categories (Figure 1, Panels, A, B, C, and D). Six patterns were discerned (Figure 2). The patterns were designated by numerical and descriptive terms as follow: pattern 1, throughout undetectable levels; pattern 2, reducing and diminishing 
Table 1: Outcomes of 101 Patients with Graves' Disease following Anti-Thyroid Drug Treatment.

\begin{tabular}{|c|c|c|c|c|c|c|}
\hline & Outcome Categories & No. of Patients & Women & Aget, Range & Men & Age, Range \\
\hline $1 a$ & Remission by attaining target $\mathrm{A}^{\dagger}$ & 41 & 31 & $9-81$ & 10 & $23-63$ \\
\hline $1 \mathbf{b}$ & Remission by attaining target $\mathrm{B}^{\dagger}$ & 11 & 10 & $30-62$ & 1 & 31 \\
\hline $2 a$ & Non-remission (attaining neither target $A$ nor $B$ ) & 38 & 28 & $24-77$ & 10 & $15-67$ \\
\hline $2 b$ & Chose thyroidectomy or radioiodine $\mathrm{Rx}^{*}$ & 6 & 3 & $21-62$ & 3 & $40-59$ \\
\hline \multirow[t]{2}{*}{3} & Developed hypothyroidism or TRAb-negative hyperthyroidism & 5 & 5 & $22-62$ & - & - \\
\hline & Total & 101 & 77 & - & 24 & - \\
\hline
\end{tabular}

*Abbreviations: ATD: Anti-Thyroid Drugs, either Thiamazole or Propypthiouracil.

Final outcome of 11 patients who relapsed after having attained Target A: one was re-treated and attained Target A again (included in outcome 1 a).

One was re-treated and attained Target B (included in outcome 1b); four were re-treated and have not attained Target A or Target B (included in outcome 2a): One opted surgery; two developed hypothyroidism, and two developed TRAb-negative hyperthyroidism (included in outcome 3).

tDefinition of terms: Target A, persistent euthyroidism with minimal doses of ATD, anti-TSH receptor antibodies by a $2^{\text {nd }}$ generation assay.

(hTRAb) titers undetectable ( $<1.0 \mathrm{IU} / \mathrm{L}$ ) on twice consecutively, and no relapse one year after ATD withdrawal; Target B, persistent euthyroidism, with minimal doses of ATD for $>6$ months, hTRAb titers continually in gray-zone $(1.0 \sim 1.5 \mathrm{IU} / \mathrm{L})$, and no relapse one year after ATD withdrawal; the age when hTRAb titers were measured first time.

into undetectable levels twice consecutively; pattern 3, reducing and converging in gray-zone levels; pattern 4, one or two flair-ups following initially reducing levels; pattern 5 , persistently high levels; pattern 6, widely fluctuating levels. The relations of outcomes and TC patterns are as follows:

\section{Panel A: Outcome 1a, remission by attaining Target $\mathrm{A}(\mathrm{N}=41)$}

The initial hTRAb levels were $<1.0-22.2 \mathrm{IU} / \mathrm{L}$ (median, $2.1 \mathrm{IU} / \mathrm{L}$ ) and $>10 \mathrm{IU} / \mathrm{L}$ in 5 patients. Another 5 patients showed pattern 1 (Figure 2) ever since they were referred to the author from nonthyroidologist physicians. They were kept on minimal dose of ATD or observed without ATD for periods for up to 13 months. Thirty-one patients $(75.6 \%, 31 / 41)$ showed TC pattern 2 . They were treated with ATD for up to 58, 36.3-58 months (median, range). TCs of other five patients $(12.2 \%, 5 / 41)$ showed pattern 4 , i.e., one episode (one patient) and two episodes (four patients) of recurrent active hyperthyroidism (flair-up) necessitating an increase in ATD doses for 20 to 47 months. Consequently, the five patients had to be treated for longer periods, 60 to 120 months.

\section{Panel B: Outcome 1b, remission by attaining Target $B(N=11)$}

The initial hTRAb levels were 2.4-53.2 IU/L (median, $5.5 \mathrm{IU} / \mathrm{L}$ ) and $>10 \mathrm{IU} / \mathrm{L}$ in 6 patients. The initial levels were higher and the reduction of the levels was slower in comparison with those of $1 \mathrm{a}$ patients. Seven patients $(63.6 \%, 7 / 11)$ showed pattern 3 and were treated with ATD for up 60 months. TCs of other four $(36.4 \%, 4 / 11)$ showed pattern 4 , i.e., flair-up following initially reducing levels, i.e., one episode (two patients) and two episodes (two patients) of flare-up of hyperthyroidism lasting 16 to 50 months. These four patients were treated for 60 to 120 months in total. Two patients were included in this outcome category despite final hTRAb titers of 1.6 and $2.2 \mathrm{IU} / \mathrm{L}$ because they had been maintained on minimal dose of ATD for more than 6 months. They did not relapse within one-year post-ATD.

\section{Panel C: Outcome 2a, unable to attain Target A or Target B and kept on ATD $(\mathrm{N}=38)$}

The initial hTRAb levels were 9.15 IU/L, <1.0-547 IU/L (median, range) and $>10 \mathrm{IU} / \mathrm{L}$ in 16 patients $(16 / 38,42.1 \%)$. The TCs are too packed in the diagram of Panel $\mathrm{C}$ to decipher their characteristics. They are divided in two small panels; Panels C' (initial hTRAb titers
$>10$ IU/L) and Panel C" (initial hTRAb titers $\leq 10 \mathrm{IU} / \mathrm{L}$ ). Three TC patterns are discerned, i.e., pattern 2 (six patients), pattern 5 (24 patients), and pattern 6 (eight patients). Four patients were included in this outcome category despite their final hTRAb levels were in the gray-zone. They reportedly had to be maintained on ATD treatment even after being transferred to other thyroidologists.

\section{Panel D: Unable to attain Target A or Target B and switch to} ablation therapy $(\mathrm{N}=6)$

The initial hTRAb levels remained elevated in the range of 4.3-42.2 IU/L in five patients. Four patients showed pattern 6 TCs and two patients showed pattern 5 . Three of them had been treated elsewhere for 120 to 302 months before being referred to the author. One patient opted for surgery because she relapsed 5 months after ATD withdrawal.

Henceforth, patients of Panels A and as B are described as "remission-likely" patients and those of Panels C and D as "remissionunlikely" patients.

\section{Discussion}

The remission rate of patients having attained either Target A or Target B was $51.5 \%$, which is comparable with that $(51.3 \%)$ of a recent meta-analysis of ATD treatment [2]. The remission rate of patients having attained Target A and no within-one-year postATD relapse (41 patients) in patients having attained Target A with or without relapse (52 patients) was $78.8 \%$. Likewise, the remission rate of combined patients having attained Targets $A$ and $B$ in those having attained Targets A and B with or without relapse was $83 \%$. These figures are better than the remission rates of patients who attain hTRAb levels below respective cutoff levels, i.e., 26.7-73.8\% (calculated from reported relapse rates) [7-13]. As for five patients of outcome 1a showing undetectable levels throughout the author's observation period, they had been treated by non-thyroidologist physicians before being referred to the author. If the present remission rate of patients attaining Target A $78.8 \%$ is probed in more patients, this would be helpful for physicians unaccustomed to ATD treatment to decide timing of ATD withdrawal.

Four patterns of TCs are found to be associated with remission, i.e., patterns 1,2,3, and 4. Michelangeli V, et al. [16], Takasu N, et al. [17], and Laurberg P, et al. [18] studied the TCs of TRAb levels 
periodically measured by a ${ }^{\text {st }}$ generation assay during ATD treatment using a titration regimen. They observed patterns 2,5 , and 6 and found pattern 2 was associated with remission.

What was observed in the present study as well as in these studies are the relations of TC patterns and the outcomes. Provided TRAb levels in blood are to reflect the status of immune dysregulation, quite the opposite, the outcomes of ATD-treated GD can be predicted by TC patterns in most situations, except for some cases (vide infra). First, it takes either 51.4 months or 74.6 months (Cf. result section) for patients to achieve relapse-free remission by attaining Targets
A or B (TC patterns 2, 3, or 4). For patients showing TC patterns 2, 3, or 4, prolonged ATD treatment appears better than life-long replacement with thyroid hormone after ablation therapy. Second, selection of "remission unlikely" patients and transferring them to ablation therapy could be facilitated by TC patterns 5 and 6 . The differentiation, however, could not be made solely by high TRAb titers in the early period, say hTRAb >10 IU/L. Periodic measurements of TRAb in the first 2 or 3 years of ATD treatment appear necessary to separate "remission unlikely" patients from "remission likely" patients. On the other hand, Hashizume K, et al. [19] and McIver B, et al. [20]

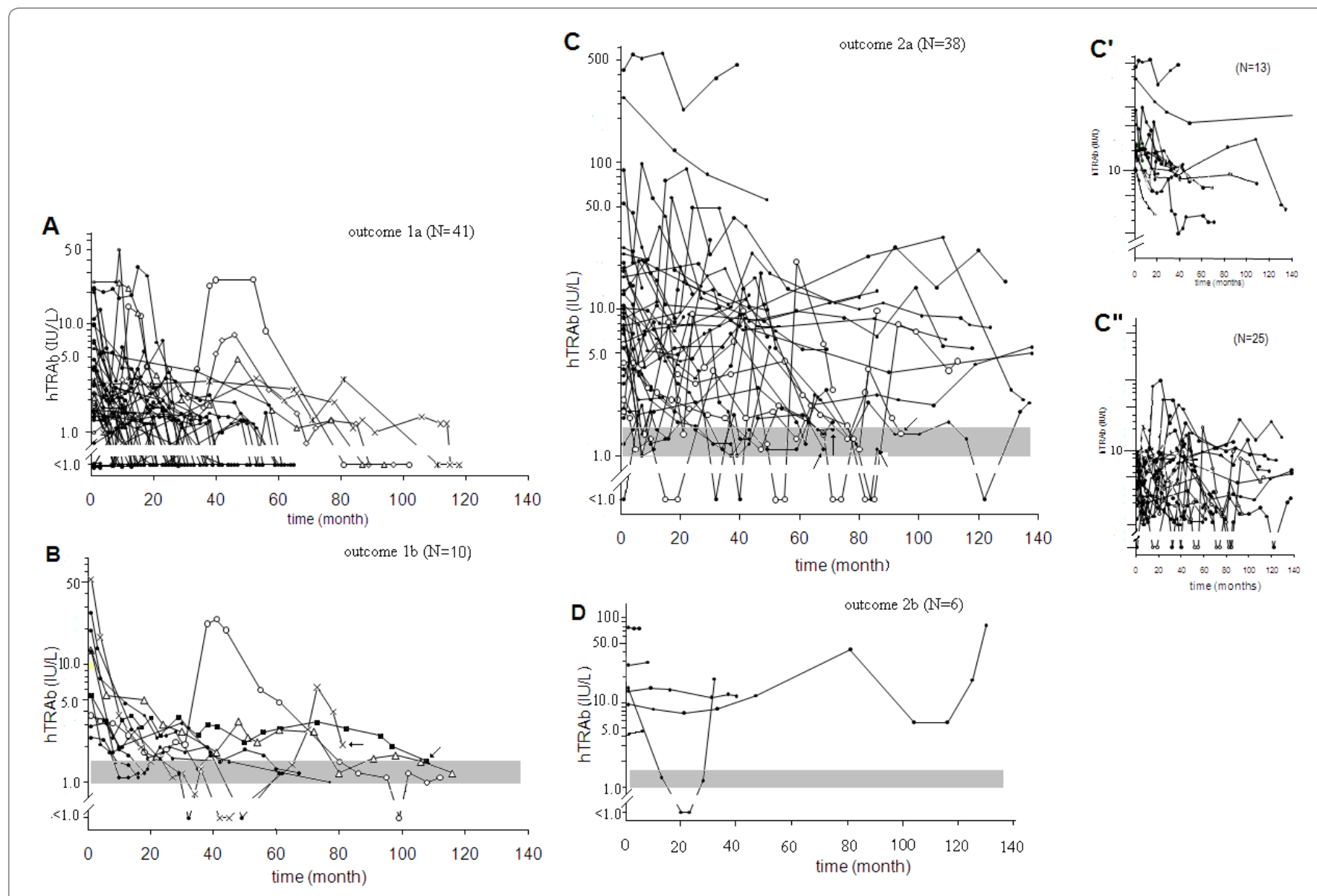

Figure 1: Time courses of titers of anti-thyrotropin receptor auto-antibodies by a $2^{\text {nd }}$ generation assay in reference assembled in outcome categories.

Time Courses (TCs) of hTRAb levels of four outcome categories are shown in four panels: Panel A, outcome 1a, disappearance of hTRAb twice with no within-one-year post-ATD relapse; Panel B, outcome 1b, hTRAb persistently in the gray-zone in the last phase of ATD with no within-one-year post-ATD relapse; Panel C, outcome 2a, hTRAb persistently at high levels or fluctuating without disappearing/converging in gray-zone; Panel D, outcome $2 b$, opted ablative therapy.

Gray-zones are shown by gray horizontal strips in Panels B, C, and D. Because the TCs of Panel C are too packed to decipher characteristics of individual TCs, these TCs were divided into two smaller panels $\left(C^{\prime}, C^{\prime \prime}\right)$. Namely, the TCs of hTRAb of patients who showed hTRAb $>10$ IU/L at the first clinic visit are shown in Panel $C^{\prime}$ and those of other patients who showed $h T R A b \leq 10 \mathrm{IU} / \mathrm{L}$ at the first clinic visit are shown in Panel $C^{\prime \prime}$.

In Panel A, data of five patients having a recurrence of hyperthyroidism accompanied by a spiked hTRAb elevation in the course are marked by $0, \diamond, \triangle, X$, and $\%$ to make their courses traceable. In Panel $B$, likewise, TCs of two patients having a recurrence of hyperthyroidism accompanied by spiked hTRAb elevation in the course are marked by $0, \triangle, \mathbf{m}$, and X. The last TRAb titers of two patients, slightly above gray-zone (1.9 and $2.1 \mathrm{lU} / \mathrm{L}$ ), are indicated by arrows. These two patients remained in euthyroidism more than 12 months after ATD withdrawal.

In Panel C, data of four patients who had relapse after ATD withdrawal are illustrated by o connected by segments. TCs are indicated by arrows in four patients in whom ATD could not be withdrawn despite last hTRAb levels were in the gray-zone.

Abbreviation: hTRAb, Anti-Thyrotropin Receptor Autoantibodies (TRAb) measured in an assay with DYNO test TRAK ${ }^{\mathrm{TM}}$ human kit. 


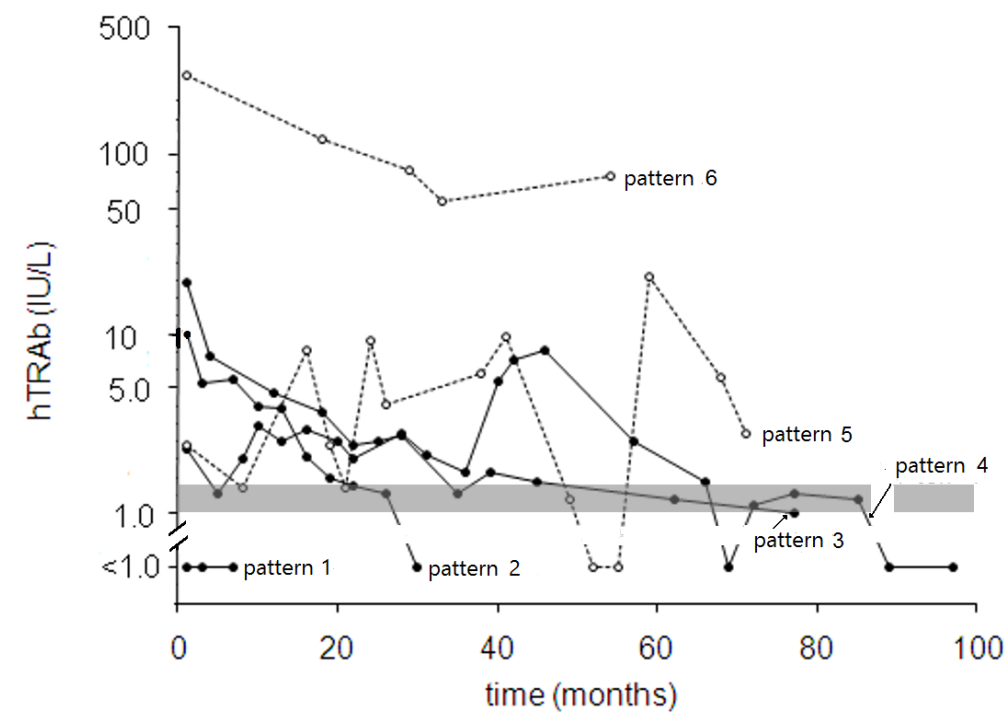

Figure 2: Six patterns of time courses during treatment with anti-thyroid drugs.

Descriptive terms of Patterns 1 through 6 are follows: Pattern 1, throughout undetectable levels; Pattern 2, reducing and diminishing in undetectable levels twice consecutively; Pattern 3, reducing and converging in gray-zone levels; Pattern 4, flair-ups following initially reducing levels; Pattern 5, persistently high levels; Pattern 6, widely fluctuating levels.

Gray-zone is shown by gray horizontal strip.

treated GD patient with ATD using a block and replace regimen, i.e., continuous administration of either methimazole $30 \mathrm{mg} /$ day [19] or carbimazole $40 \mathrm{mg}$ /day [20] for the 18 months and replacing with L-thyroxine when hypothyroidism is induced by high doses of ATD. They followed TRAb levels by a $2^{\text {nd }}$ generation assay and observed progressive reduction of TRAb levels in all patients. Maintaining of high ATD doses in the early phase of ATD treatment might alter the immune dysregulation of GD, which need to be confirmed in larger studies. Third, nine patients experienced one or two episodes of flareup of hyperthyroidism despite faithful adherence to ATD treatment for 2 to 5 years. Takasu N [17] and coworkers also observed the flair-ups of hyperthyroidism in a few patients who showed gradually declining TRAb levels in the initial period of ATD treatment. Hidaka Y [21] and coworkers reported flare-up of hyperthyroidism accompanied by TRAb rise concurrently with seasonal exacerbation of allergic rhinitis. Cigarette smoking was reported to be associated with an increased recurrence rate after withdrawal of ATD [22]. Histories of smoking and allergic rhinitis, however, were not elicited in the present patients who experienced flare-ups. Clues to driving forces of perpetuating hyperthyroidism and flare-ups could be obtained by investigating clinical and immunological factors associated by three TC patterns, patterns 4,5 , and 6 .

ATD was continued prospectively until hTRAb levels become undetectable consecutively twice or remain in gray-zone, presuming hTRAb level is a surrogate biomarker of disordered immune status. There were, however, a few exceptions to this presumption. First, the TRAb assay employed does not differentiate TSH receptor blocking activity and TSH-receptor stimulating activity, e.g., one patient became hypothyroid in the presence of high hTRAb levels and her hTRAb supposedly had TSH receptor blocking activity. Of the four patients of outcome 3, two developed hypothyroidism and other two remained in mild hyperthyroidism despite repetitively undetectable hTRAb levels. Undetectable hTRAb levels of these patients may not necessarily mean a thorough absence of anti-TSH receptor auto-antibodies in the blood as well as in the thyroid tissue. Second, two patients having been maintained on minimal dose of ATD for $>$ six months were included in outcome $1 \mathrm{~b}$ despite final hTRAb levels slightly above gray-zone, 1.6 and 2.2 IU/L, respectively. They had not have relapse after ATD withdrawal. Third, four patients were included in outcome 2a (nonremission) despite their final hTRAb levels in the gray-zone. The four reportedly had to be maintained on ATD treatment after being transferred to other thyroidologists. As TRAb levels during ATD treatment reflect the disease activity of GD in the majority of cases $(89.1 \%, 90 / 101)$, hTRAb levels can used as a biomarker of the status of immune dysregulation in selected GD patients during ATD treatment until better and inexpensive markers become available.

Lastly, $3^{\text {rd }}$ generation competition-based TSH receptor antibody assay is available since 2008, which utilizes labeled monoclonal human TSH receptor antibody as a competitive ligand. This $3^{\text {rd }}$ generation assay, however, has reportedly not necessarily improved the prediction of remission in comparison with the $2^{\text {nd }}$ generation assay [10]. In parallel with the improvement of competition-based TRAb assays, receptor-stimulating TRAb (TSAb) assays have been improved [23]. The $3^{\text {rd }}$ generation TSAb assay measures TSH stimulating antibodies using Chinese hamster ovary Mc4 cell, in which 73 amino acids of A segment of TSH receptor containing the epitope for binding with receptor-blocking TRAb was substituted with 73 amino acids of rat LH receptor (chimeric human TSH receptor) [24]. With the $3^{\text {rd }}$ generation TSAb assay. Kwon $\mathrm{H}$, et al. [13] examined the relation of relapse and TRAb status in 35 patients and found relapse rates in patients with positive and negative TSAb were $67 \%(8 / 12)$ and $17 \%$ $(4 / 23)$, respectively. The $3^{\text {rd }}$ generation TSAb assay especially useful to assess the status of disordered immune status of GD during ATD treatment. Their finding needs to be are confirmed in larger number of patients. 


\section{Conclusion}

Though ATD have been employed for GD for years worldwide, remission rate of GD by ATD is still not satisfactory. As GD is an autoimmune disease caused by TRAb, treatment preferably is to follow bio-markers reflecting disordered immune status. TRAb levels measured by the present assay do not necessarily accurately reflect the immune status in every patient. Nonetheless, TCs of hTRAb levels match with the outcomes of GD in many patients. TCs could be used for determine prolonged ATD or ablation therapy. The investigation into clinical and immunological findings associated with flair-ups during ATD treatment, widely fluctuating TRAb levels, and persistently high TRAb levels may provide clues to detect underlying causes of these manifestations.

\section{Acknowledgements}

The author declares no conflicts of interest in this report. In the author's country, the cost of hTRAb is stipulated nation-wide as $\$ 31.8$ (based on current exchange rate) according to the country's health insurance system. Patients have to pay 10 or $30 \%$ of this cost and the rest paid by the national health insurance system. Hence, the cost of TRAb pointed out by Mathews DC \& Syed AA (Eur J Int Med 2011; $22: 213$ ) is not an obstacle.

\section{References}

1. Astwood EB (1943) Treatment of hyperthyroidism with thiourea and thiouracil. JAMA 251: 1743-1746.

2. Struja T, Fehlberg H, Kutz A, Guebelin L, Degen C, et al. (2017) Can we predict relapse in Graves' disease? Results from a systematic review and meta-analysis. Eur J Endocrinol 176: 87-97.

3. Ross D, Burch HB, Cooper DS, Greenlee MC, Laurberg P, et al. (2016) 2016 American Thyroid Association guidelines for diagnosis and management of hyperthyroidism and other causes of thyrotoxicosis. Thyroid 26: 1343-1421.

4. Costagliola S, Morgenthaler NG, Hoermann R, Badenhoop K, Struck $J$, et al. (1999) Second generation assay for thyrotropin receptor antibodies has superior diagnostic sensitivity for Graves' disease. J Clin Endocrinol Metab 84: 90-97.

5. Kamijo K (2003) TSH-receptor antibody measurement in patients with various thyrotoxicosis and Hashimoto's thyroiditis: a comparison of two two-step assays, coated plate ELISA using porcine TSH-receptor and coated tube radioassay using human recombinant TSH-receptor. Endocr J 50: 113-116.

6. Barbesino G, Tomer Y (2013) Clinical review: Clinical utility of TSH receptor antibodies. J Clin Endocrinol Metab 98: 2247-2255.

7. Zimmermann-Belsing T, Nygaard B, Rasmussen AK, Feldt-Rasmussen $U$ (2002) Use of the $2^{\text {nd }}$ generation TRAK human assay did not improve prediction of relapse after antithyroid medical therapy of Graves' disease. Eur J Endocrinol 146: 173-177.

8. Schott M, Morgenthaler NG, Fritzen R, Feldkamp J, Willenberg HS, et al. (2004) Levels of autoantibodies against human TSH receptor predict relapse of hyperthyroidism in Graves' disease. Horm Metab Res 36: 92-96.

9. Okamoto $Y$, Tanigawa S, Ishikawa K, Hamada N (2006) TSH receptor antibody measurements and prediction of remission in Graves disease patients treated with minimal maintenance doses of antithyroid drugs. Endocr J 53: 467-472.

10. Massart C, Gibassier J, d'Herbonez M (2009) Clinical value of M22based assays for TSH-receptor antibody (TRAb) in the follow-up of antithyroid drug treated Graves' disease: comparison with the second generation human TRAb assay. Clin Chim Acta 407: 62-66.

11. Konishi T, Okamoto Y, Ueda M, Fukada Y, Harusato I, et al. (2011) Drug discontinuation after treatment with minimal maintenance dose of an antithyroid drug in Graves' disease: a retrospective study on effects of treatment duration with minimum maintenance dose on lasting remission. Endocr J 58: 95-100.

12. Tun NN, Beckell G, Zammitt NN, Strachen MW, Seckl JR, et al. (2016) Thyrotropin receptor antibody levels at diagnosis and after thionamide course predict Graves' disease relapse. Thyroid 26: 1004-1009.

13. Kwon H, Kim WG, Jang EK, Kim M, Park S, et al. (2016) Usefulness of measuring thyroid stimulating antibody at the time of antithyroid drug withdrawal for predicting relapse of Graves' disease. Endocrinol Metab (Seoul) 31: 300-310.

14. Liu L, Lu H, Liu Y, Liu C, Xun C (2016) Predicting relapse of Graves' disease following treatment with antithyroid drugs. Exp Ther Med 11: $1453-1458$

15. Japan Thyroid Association (2011) Drug treatment. In: Nakamura $\mathrm{H}$, Konishi J (eds) Guideline for the treatment of Graves' disease in Japan. Japan 75-95.

16. Michelangeli V, Poon C, Taft J, Newnham H, Topliss D, et al. (1998) The prognostic value of thyrotropin receptor antibody measurement in the early stages of treatment of Graves' disease with antithyroid drugs. Thyroid 8: 119-124.

17. Takasu N, Yamashiro K, Komiya I, Ochi Y, Sato Y, et al. (2000) Remission of Graves' hyperthyroidism predicted by smooth decreases of thyroid-stimulating antibody and thyrotropin-binding inhibitor immunoglobulin during antithyroid drug treatment. Thyroid 10: 891-896.

18. Laurberg P, Wallin G, Tallstedt L, Abraham-Nording M, Lundell G, et al. (2008) TSH-receptor autoimmunity in Graves' disease after therapy with anti-thyroid drugs, surgery, or radioiodine: a 5-year prospective randomized study. Eur J Endocrinol 158: 69-75.

19. Hashizume K, Ichikawa K, Sakurai A, Suzuki S, Takeda T, et al. (1991) Administration of thyroxine in treated Graves' disease: effects on the level of antibodies to thyroid-stimulating hormone receptors and on the risk of recurrence of hyperthyroidism. N Engl J Med 324: 947-953.

20. Mclver B, Rae P, Beckett G, Wilkinson E, Gold A, et al. (1996) Lack of effect of thyroxine in patients with Graves' hyperthyroidism who are treated with an antithyroid drug. N Engl J Med 334: 220-224.

21. Hidaka $Y$, Amino N, Iwatani $Y$, Itoh E, Matsunaga $M$, et al. (1993) Recurrence of thyrotoxicosis after attack of allergic rhinitis in patients with Graves' disease. J Clin Endocrinol Metab 77: 16671670.

22. Glinoer D, de Nayer P, Bex M, Belgian Collaborative Study Group on Graves' Disease (2001) Effects of L-thyroxine administration, TSH-receptor antibodies and smoking on the risk of recurrence in Graves' hyperthyroidism treated with antithyroid drugs: a doubleblind prospective randomized study. Eur J Endocrinol 144: 475-483.

23. Hesarghatta Shyamasunder A, Abraham P (2017) Measuring TSH receptor antibody to influence treatment choices in Graves' disease. Clin Endocrinol (Oxf) 86: 652-657.

24. Lytton SD, Ponto KA, Kanitz M, Matheis N, Kohn LD, et al. (2010) A novel thyroid stimulating immunoglobulin bioassay is a functional indicator of activity and severity of Graves' orbitopathy. J Clin Endocrinol Metab 95: 2123-2131. 